\title{
GLOBAL STRONG SOLUTIONS OF EQUATIONS OF MAGNETOHYDRODYNAMIC TYPE
}

\author{
MARKO A. ROJAS-MEDAR ${ }^{1}$ and JOSÉ LUIZ BOLDRINI ${ }^{1}$
}

(Received 1 November 1993; revised 14 June 1995)

\begin{abstract}
By using the spectral Galerkin method, we prove a result on the global existence in time of strong solutions for a system of equations of magnetohydrodynamic type. Several estimates for the solution and their approximations are given. These estimates can be used in the derivation of error bounds for the approximate solutions.
\end{abstract}

\section{Introduction}

In several situations the motion of incompressible electrical conducting fluids can be modelled by the so-called equations of magnetohydrodynamics, which correspond to the Navier-Stokes equations coupled with Maxwell's equations. In the case where there is free motion of heavy ions, not directly due to the electric field (see $[10,11,9]$ ), these equations can be reduced to the form

$$
\left\{\begin{array}{l}
\frac{\partial u}{\partial t}-\frac{\eta}{\rho_{m}} \Delta u+u \cdot \nabla u-\frac{\mu}{\rho_{m}} h \cdot \nabla h=f-\frac{1}{\rho_{m}} \nabla\left(p^{*}+\frac{\mu}{2} h^{2}\right), \\
\frac{\partial h}{\partial t}-\frac{1}{\mu \sigma} \Delta h+u \cdot \nabla h-h \cdot \nabla u=-\nabla w, \\
\operatorname{div} u=0, \quad \operatorname{div} h=0, \\
\left.u\right|_{\partial \Omega}=0,\left.\quad h\right|_{\partial \Omega}=0, \\
\left.u\right|_{t=0}=u_{0},\left.\quad h\right|_{t=0}=h_{0} .
\end{array}\right.
$$

In these equations we have assumed homogeneous boundary conditions just for simplicity. In standard ways, the nonhomogeneous case could be treated by imposing customary conditions on the boundary data.

'UNICAMP-IMECC, CP. 6065, 13081-970, Campinas, SP, Brazil

(C) Australian Mathematical Society, 1997, Serial-fee code 0334-2700/96 
Here, $u$ and $h$ are respectively the unknown velocity and magnetic fields; $p^{*}$ is the unknown hydrostatic pressure; $w$ is an unknown function related to the motion of heavy ions (in such way that the density of electric current, $j_{0}$, generated by this motion satisfies the relation rot $\left.j_{0}=-\sigma \nabla w\right) ; \rho_{m}$ is the density of mass of the fluid (assumed to be a positive constant); $\mu>0$ is the constant magnetic permeability of the medium; $\sigma>0$ is the constant electric conductivity; $\eta>0$ is the constant viscosity of the fluid; $f$ is a given external force field.

The stationary problem corresponding to (1.1) was considered by Chizhonkov [3], while the question of the (local) existence of a solution of the evolution problem (1.1) was analysed by Lassner [7], making use of semigroup techniques similar to ones in Fujita and Kato [5] (who also studied the asymptotic behavior of the solution as $t \rightarrow 0^{+}$). The more constructive spectral Galerkin method was used by Boldrini and Rojas-Medar [2] to obtain local-time strong solutions.

In this paper we consider the problem of the global existence of strong solutions of (1.1), with homogeneous boundary conditions for $u$ and $h$ for simplicity of exposition. The spectral Galerkin method of approximation will be used. Thus, the results in this paper form the theoretical basis for future numerical analysis of the problem: here we shall obtain estimates for the approximate solutions that will be fundamental in a forthcoming paper in which optimal uniform error estimates for such Galerkin approximations will be obtained.

The paper is organized as follows. In Section 2 the basic assumptions and results that will be used later in the paper are stated. We also rewrite (1.1) in a more suitable weak form, describe the approximation method and state the results of the paper (Theorems 4, 5, 6 and 7). The following sections will be devoted to their proofs.

\section{Preliminaries and results}

Let $\Omega \subseteq \mathbb{R}^{n}, n=2$ or 3 , be a bounded domain with boundary $\partial \Omega$ of class $C^{1,1}$. Denote by $H^{s}(\Omega)$ the Sobolev spaces on $\Omega$ with norm $\|\cdot\|_{s},(\cdot, \cdot)$ denoting the $L^{2}$ norm on $\Omega$. $H_{0}^{1}(\Omega)$ is the completion of $C_{0}^{\infty}(\Omega)$ under the norm $\|\cdot\|_{1}$. Also, denote by $L^{p}(\Omega)$ for $1 \leq p \leq \infty$ the usual Lebesgue spaces and by $\|\cdot\|_{L^{p}}$ the $L^{p}$-norm on $\Omega$. With the same symbols denote the spaces of $n$-dimensional vector functions. If $B$ is a Banach-space, denote by $L^{q}([0, T) ; B)$ the Banach space of the $B$-valued functions defined in the interval $[0, T)$ that are $L^{q}$-integrable in the sense of Bochner and by $L_{l o c}^{q}([0, T) ; B)$ the $B$-valued functions defined in the interval $[0, T)$ that are $L^{q}$-integrable in $[0, a]$, for any $a<T \leq+\infty$. 
We put

$$
\begin{aligned}
C_{0, \sigma}^{\infty}(\Omega) & =\left\{v \in C_{0}^{\infty}(\Omega) / \operatorname{div} v=0\right\} \\
H & =\text { closure of } C_{0, \sigma}^{\infty}(\Omega) \text { in }\left(L^{2}(\Omega)\right)^{n} \\
V & =\text { closure of } C_{0, \sigma}^{\infty}(\Omega) \text { in }\left(H^{1}(\Omega)\right)^{n} .
\end{aligned}
$$

It is possible to show that

$$
V=\left\{v \in H_{0}^{1}(\Omega) \mid \operatorname{div} v=0\right\}
$$

Recall the Helmholtz decomposition of a vector field: $\left(L^{2}(\Omega)\right)^{n}=H \oplus G$, where $G=\left\{\phi \mid \phi=\nabla p, p \in H^{l}(\Omega)\right\}$.

Throughout the paper $P$ will denote the orthogonal projection from $L^{2}(\Omega)$ onto $H$. The operator $A: H \rightarrow H$ given by $A=-P \Delta$ with domain $D(A)=\left(H^{2}(\Omega)\right)^{n} \cap V$ is called the Stokes operator. It is well known that $A$ is a positive definite self-adjoint operator and it is characterized by the relation

$$
(A w, v)=(\nabla w, \nabla v) \quad \text { for all } \quad w \in D(A), v \in V
$$

Observe that for the regularity properties of the Stokes operator, it is usually assumed that $\Omega$ is of class $C^{3}$, this being in order to use Cattabriga's results $[4,12]$. We use instead the stronger results of Amrouche and Girault [1] which imply, in particular, that when $A u \in L^{2}(\Omega)$ then $u \in H^{2}(\Omega)$ and $\|u\|_{H^{2}}$ and $\|A u\|$ are equivalent norms when $\Omega$ is of class $C^{1,1}$.

The operator $A^{-1}$ is linear continuous from $H$ into $D(A)$, and, since the injection of $D(A)$ in $H$ is compact, $A^{-1}$ can be considered as a compact operator in $H$. As an operator in $H, A^{-1}$ it is also self-adjoint. By a well-known theorem of Hilbert, there exists a sequence of positive numbers $\mu_{j}>0, \mu_{j+1} \leq \mu_{j}$ and an orthonormal basis of $H,\left(w_{j}\right)$, such that $A^{-1} w_{j}=\mu_{j} w_{j}$. We put $\lambda_{j}=\mu_{j}^{-1}$. Since $A^{-1}$ has its range in $D(A)$ we obtain that $A w_{j}=\lambda_{j} w_{j}, w_{j} \in D(A), 0<\lambda_{1}<\ldots \leq \lambda_{j} \leq \lambda_{j+1} \leq \ldots$ and $\lim _{j \rightarrow+\infty} \lambda_{j}=+\infty$. Also, $\left\{w_{j}\right\}_{j=1}^{\infty}$ is an orthonormal basis for $H$ and $\left\{w_{j} / \sqrt{\lambda_{j}}\right\}_{j=1}^{\infty}$ and $\left\{w_{j} / \lambda_{j}\right\}_{j=1}^{\infty}$ form an orthonormal basis in $V$ (with the inner product $(\nabla u, \nabla v), u, v \in V$ ) and $H^{2}(\Omega) \cap V$ (with the inner product $(A u, A v), u, v \in D(A)$, respectively).

By using the properties of $P$, we can reformulate the problem (1.1), with homogeneous boundary conditions, as follows: find $u, h$, in suitable spaces to be exactly defined later on, satisfying

$$
\left\{\begin{array}{l}
\alpha\left(u_{t}, \phi\right)+\alpha(u . \nabla u, \phi)-(h . \nabla h, \phi)+v(A u, \phi)=(\alpha f, \phi), \\
\left(h_{t}, \psi\right)+(u . \nabla h, \psi)-(h . \nabla u, \psi)+\gamma(A h, \psi)=0 \\
\text { for } \quad 0<t<T, \forall \phi, \psi \in V \text { and } \\
u(0)=u_{0}, h(0)=h_{0} .
\end{array}\right.
$$


Here we have $\alpha=\rho_{m} / \mu, \nu=\eta / \mu, \gamma=1 /(\mu \sigma)$.

Now we define strong solutions of the problem (2.1).

DEFINITION. Let $u_{0}, h_{0} \in V$ and $f \in L^{\infty}\left([0, \infty) ; L^{2}(\Omega)\right)$. By a strong solution of the problem (2.1), we mean a pair of vector-valued functions $(u, h)$ that satisfies $(2.1)$ and such that $u, h \in L^{\infty}([0, \infty) ; V) \cap L_{l o c}^{2}([0, \infty) ; D(A))$.

REMARK. In what follows, we prove that if $(u, h)$ is a strong solution of $(2.1)$ then $u_{t}, h_{t} \in L_{l o c}^{2}([0, \infty] ; H)$. This condition, together with $u, h \in L_{l o c}^{2}([0, \infty) ; D(A))$, implies by interpolation (see [12, p. 260]) that $u, h$ are almost everywhere equal to a continuous function from $[0, T]$ into $V(0<T<+\infty)$. Consequently, the initial conditions $u(0)=u_{0}$ and $h(0)=h_{0}$ are meaningful.

To prove existence of strong solutions we apply the spectral Galerkin method to (2.1). That is, we consider the finite-dimensional subspaces $V_{k}=\operatorname{span}\left[w^{1}, \ldots, w^{k}\right]$, $k \in \mathbb{N}$, the corresponding orthogonal projections $P_{k}: H \rightarrow V_{k}$ and the approximate solutions

$$
u^{k}(x, t)=\sum_{i=1}^{k} c_{i k}(t) w^{i}(x), \quad h^{k}(x, t)=\sum_{i=1}^{k} d_{i k}(t) w^{i}(x),
$$

developed in terms of eigenfunctions of the Stokes operator. Then, the coefficients $c_{i k}(t)$ and $d_{i k}(t)$ are found by requiring that $u^{k}$ and $h^{k}$ satisfy the following equations:

$$
\left\{\begin{array}{l}
\alpha u_{t}^{k}+v A u^{k}+\alpha P_{k}\left(u^{k} . \nabla u^{k}\right)-P_{k}\left(h^{k} . \nabla h^{k}\right)-\alpha P_{k} f=0, \\
h_{t}^{k}+\gamma A h^{k}+P_{k}\left(u^{k} . \nabla h^{k}\right)-P_{k}\left(h^{k} . \nabla u^{k}\right)=0, \\
u^{k}(0)=P_{k} u_{0}, h^{k}(0)=P_{k} h_{0} .
\end{array}\right.
$$

This is equivalent to the weak form

$$
\left\{\begin{array}{l}
\alpha\left(u_{t}^{k}, \phi\right)+v\left(\nabla u^{k}, \nabla \phi\right)+\alpha\left(u^{k} . \nabla u^{k}, \phi\right)-\left(h^{k} . \nabla h^{k}, \phi\right)=\alpha(f, \phi), \\
\left(h_{t}^{k}, \psi\right)+\gamma\left(\nabla h^{k}, \nabla \psi\right)+\left(u^{k} . \nabla h^{k}, \psi\right)-\left(h^{k} . \nabla u^{k}, \psi\right)=0, \\
u^{k}(0)=P_{k} u_{0}, h^{k}(0)=P_{k} h_{0}, \quad \forall \phi, \psi \in V_{k} .
\end{array}\right.
$$

By using these approximations, Boldrini and Rojas-Medar [2] proved a local-time existence theorem for (2.1). Their results are the following.

PRoposition 1. Let the initial values $u_{0}, h_{0} \in V$ and the external force $f \in L^{2}(0, T$; $\left.\left(L^{2}(\Omega)\right)^{n}\right)$. Then, on a (possibly small) time interval $\left[0, T_{1}\right], 0<T_{1} \leq T$, problem (2.1) has a unique strong solution $(u, h)$. This solution belongs to. $C\left(\left[0, T_{1}\right], V\right)$. 
Moreover, there exist continuous functions $F$ and $G$ such that for any $t \in\left[0, T_{1}\right]$,

$$
\|\nabla u(t, \cdot)\|^{2}+\|\nabla h(t, \cdot)\|^{2}+\int_{0}^{t}\left(\|A u(\tau, \cdot)\|^{2}+\|A h(\tau, \cdot)\|^{2}\right) d \tau \leq F(t)
$$

and

$$
\int_{0}^{t}\left(\left\|u_{t}(\tau, \cdot)\right\|^{2}+\left\|h_{t}(\tau, \cdot)\right\|^{2}\right) d \tau \leq G(t) .
$$

Also, the same kind of estimates hold uniformly in $k \in \mathbb{N}$ for the Galerkin approximations $\left(u^{k}, h^{k}\right)$.

PROPOSITION 2. In addition to the assumptions of Proposition 1, assume that $u_{0}, h_{0} \in$ $V \cap\left(H^{2}(\Omega)\right)^{n}$ and that $f_{t} \in L^{2}\left(0, T ;\left(L^{2}(\Omega)\right)^{n}\right)$. Then, the functions $u, h$ satisfy

$$
\begin{aligned}
&\left\|u_{t}(t, \cdot)\right\|^{2}+\left\|h_{t}(t, \cdot)\right\|^{2}+ \int_{0}^{t}\left(\left\|\nabla u_{t}(\tau, \cdot)\right\|^{2}+\left\|\nabla h_{t}(\tau, \cdot)\right\|^{2}\right) d \tau \leq H(t) \\
&\|A u(t, \cdot)\|^{2}+\|A h(t, \cdot)\|^{2} \leq L(t) ; \\
& \int_{0}^{t}\left(\left\|u_{t t}(\tau, \cdot)\right\|_{V^{*}}^{2}+\left\|h_{t t}(\tau, \cdot)\right\|_{V^{*}}^{2}\right) d \tau \leq M(t),
\end{aligned}
$$

for everyt $\in\left[0, T_{1}\right]$, where $H(\cdot), L(\cdot)$ and $M(\cdot)$ are continuous functions int $\in\left[0, T_{1}\right]$. Also, $u, h \in C^{1}\left(\left[0, T_{1}\right] ; H\right) \cap C\left(\left[0, T_{1}\right], D(A)\right)$.

Moreover, the same kind of estimates hold uniformly in $k$ for the Galerkin approximations $\left(u^{k}, h^{k}\right)$.

As a consequence of the above, using the results of Amrouche and Girault [1], we conclude the following.

PROPOSITION 3. Under the hypothesis of Proposition 1, there exist unique functions $p, w \in L^{2}\left(0, T ; H^{1}(\Omega) / \mathbb{R}\right)$ such that when $p^{*}=p-\frac{\mu}{2} h^{2},\left(u, h, p^{*}, w\right)$ satisfies (1.1). Under the hypothesis of Proposition 2, $p, w \in L^{\infty}\left(0, T_{1} ; H^{1}(\Omega) / \mathbb{R}\right) \cap$ $C\left(\left[0, T_{1}\right], L^{2}(\Omega) / \mathbb{R}\right)$.

Now we state the results that will be proved in this paper.

THEOREM 4. Suppose that $n=3$, that $u_{0}, v_{0} \in V$ and that $f \in L^{\infty}\left([0, \infty) ;\left(L^{2}(\Omega)\right)^{n}\right)$. If $\left\|u_{0}\right\|_{V},\left\|h_{0}\right\|_{V}$ and $\|f\|_{L^{\infty}\left([0, \infty): L^{2}(\Omega)\right)}$ are sufficiently small, then the solution $(u, h)$ of problem (2.1) exists globally in time and satisfies $u \in C([0, \infty), V)$. Moreover, 
for any $\theta>0$ there exists some finite positive constants $M$ and $C$ such that

$$
\begin{aligned}
& \sup _{t \geq 0}\{\|\nabla u(t)\|,\|\nabla h(t)\|\}=M, \\
& \sup _{t \geq 0} e^{-\theta t} \int_{0}^{t} e^{\theta s}\left\{\left\|u_{t}(s)\right\|^{2}+\left\|h_{t}(s)\right\|^{2}\right\} d s \leq C, \\
& \sup _{t \geq 0} e^{-\theta t} \int_{0}^{t} e^{\theta s}\left\{\|A u(s)\|^{2}+\|A h(s)\|^{2}\right\} d s \leq C .
\end{aligned}
$$

Also, the same kind of estimates hold uniformly in $k$ for the Galerkin approximations.

REMARK. We have distinguished the constant in the first of the above estimates because it will play a special role in many points in the arguments that follow.

THEOREM 5. Under the hypothesis of Theorem 4 and in addition $u_{0}, h_{0} \in V \cap H^{2}(\Omega)$ and $f_{l} \in L^{\infty}\left([0, \infty) ;\left(L^{2}(\Omega)\right)^{n}\right)$, the solution obtained in Theorem 4 satisfies

$$
u \in C\left([0, \infty) ;\left(H^{2}(\Omega)\right)^{n} \cap V\right) \cap C^{1}([0, \infty) ; H) .
$$

Moreover, for any $\theta>0$ there exists one finite positive constant $C$ such that

$$
\begin{aligned}
& \sup _{t \geq 0}\left\{\left\|u_{t}\right\|,\left\|h_{t}\right\|\right\} \leq C, \\
& \sup _{t \geq 0}\{\|A u\|,\|A h\|\} \leq C, \\
& \sup _{t \geq 0} e^{-\theta t} \int_{0}^{t} e^{\theta s}\left(\left\|\nabla u_{t}(s)\right\|^{2}+\left\|\nabla h_{t}(s)\right\|^{2}\right) d s \leq C, \\
& \sup _{t \geq 0} e^{-\theta t} \int_{0}^{t} e^{\theta s}\left(\left\|u_{t t}(s)\right\|_{V^{*}}^{2}+\left\|h_{t t}(s)\right\|_{V^{*}}^{2}\right) d s \leq C .
\end{aligned}
$$

Also, the same kind of estimates hold uniformly in $k$ for the Galerkin approximations.

For the system (2.1) it is possible to recover the classical result, that usually holds for the Navier-Stokes equations, that in the two-dimensional case it is not necessary to assume the smallness of the initial data and external force. In fact, we have as follows.

THEOREM 6. Suppose that $n=2$, that $u_{0}, h_{0} \in V$ and that $f \in L^{\infty}\left([0, \infty) ;\left(L^{2}(\Omega)\right)^{n}\right)$. Then the solution $(u, h)$ of problem (2.1) exists globally in time and satisfies $u \in$ $C([0, \infty) ; V)$. Moreover, the estimates (2.4)-(2.6) are true for any $\theta>0$. 
THEOREM 7. Under the hypothesis of Theorem 6 and if in addition $u_{0}, h_{0} \in V \cap$ $\left(H^{2}(\Omega)\right)^{n}$ and $f_{t} \in L^{\infty}\left([0, \infty) ;\left(L^{2}(\Omega)\right)^{n}\right)$, then the solution $(u, h)$ satisfies

$$
u, h \in C\left([0, \infty) ; V \cap\left(H^{2}(\Omega)\right)^{n}\right) \cap C^{1}([0, \infty) ; H) .
$$

Moreover, the estimates (2.7)-(2.10) are true for any $\theta>0$.

As a consequence of the above, using the results of Amrouche and Girault [1], we conclude the following.

THEOREM 8. Under the hypothesis of Theorem 4 or 6 , there exist unique functions $p, w \in L_{\text {loc }}^{2}\left(0, \infty ; H^{1}(\Omega) / \mathbb{R}\right)$ such that when $p^{*}=p-\frac{\mu}{2} h^{2},\left(u, h, p^{*}, w\right)$ is a solution of (1.1) and satisfies for any $\theta>0$ the condition

$$
\sup _{t \geq 0} e^{-\theta t} \int_{0}^{t} e^{\theta s}\left(\|p\|_{H^{\prime}(\Omega) / \mathbb{R}}^{2}+\|w\|_{H^{1}(\Omega) / \mathbb{R}}^{2}\right) d s<+\infty .
$$

Under the hypothesis of Theorem 5 or $7, p, w \in L^{\infty}\left(0, \infty ; H^{1}(\Omega) / \mathbb{R}\right) \cap C([0, \infty)$; $\left.L^{2}(\Omega) / \mathbb{R}\right)$ and satisfy

$$
\sup _{i \geq 0}\left\{\|p\|_{H^{\prime}(\Omega) / \mathbb{R}}^{2}+\|w\|_{H^{\prime}(\Omega) / \mathbb{R}}^{2}\right\}<+\infty .
$$

\section{The proofs of Theorems 4 and 5}

We start by proving the boundedness in time of $\|\nabla u(t)\|,\|\nabla h(t)\|$. From Boldrini and Rojas-Medar [2, p. 8], we have the differential inequality

$$
\frac{d}{d t}\left(\alpha\|\nabla u\|^{2}+\|\nabla h\|^{2}\right)+v\|A u\|^{2}+\gamma\|A h\|^{2} \leq C_{1}\left(\alpha^{5}\|\nabla u\|^{10}+\|\nabla h\|^{10}\right)+C_{3},
$$

where $C, C_{1}$ and $C_{3}=C \sup _{t \geq 0}\|f(t)\|$ are positive constants. Now, observe that there exist $C_{4}>0, C_{5}>0$ such that

$$
\begin{aligned}
C_{4} \alpha\|\nabla u\|^{2} & \leq v\|A u\|^{2}, \\
C_{5}\|\nabla h\|^{2} & \leq \gamma\|A h\|^{2},
\end{aligned}
$$

So, inequality (3.1) becomes

$$
\begin{aligned}
\frac{d}{d t}\left(\alpha\|\nabla u\|^{2}+\|\nabla h\|^{2}\right) & \leq C_{1}\left(\alpha^{5}\|\nabla u\|^{10}+\|\nabla h\|^{10}\right)-C_{4} \alpha\|\nabla u\|^{2}-C_{5}\|\nabla h\|^{2}+C_{3} \\
& \leq C_{1}\left(\alpha^{5}\|\nabla u\|^{10}+\|\nabla h\|^{10}\right)-C_{2}\left(\alpha\|\nabla u\|^{2}+\|\nabla h\|^{2}\right)+C_{3},
\end{aligned}
$$


where $C_{2}>0$.

Letting $\psi(t)=\alpha\|\nabla u(t)\|^{2}+\|\nabla h(t)\|^{2}$ produces the differential inequality

$$
\begin{gathered}
\frac{d \psi}{d t} \leq C_{1} \psi^{5}-C_{2} \psi+C_{3}, \\
\psi(0)=\alpha\left\|\nabla u_{0}\right\|^{2}+\left\|\nabla h_{0}\right\|^{2} .
\end{gathered}
$$

We consider the corresponding differential equation

$$
\begin{aligned}
\frac{d \phi}{d t} & =C_{1} \phi^{5}-C_{2} \phi+C_{3}=F\left(C_{3}, \phi\right), \\
\phi(0) & =\psi(0) .
\end{aligned}
$$

Results from the differential inequalities imply $\psi(t) \leq \phi(t)$ for all $t$ in the interval of existence. Observe that, when $C_{3}=0, F(0, \phi)=C_{1} \phi^{5}-C_{2} \phi$. Consequently, $F(0, \phi)$ has a simple root given by $r(0)=\left(C_{2} / C_{1}\right)^{1 / 4}$. This root is unstable, and so, for $C_{3}$ small, $F\left(C_{3}, \phi\right)$ also has one unstable simple root $r\left(C_{3}\right)$ close to $r(0)$. Thus, if $0<\psi(0)=\phi(0)=r\left(C_{3}\right)$, we have $0 \leq \psi(t) \leq \phi(t) \leq r\left(C_{3}\right)<+\infty$ for all $t$ in the interval of existence. Thus, there exists a constant $M>0$ such that

$$
\sup _{i \geq 0}\{\|\nabla u\|,\|\nabla h\|\}=M<+\infty .
$$

Now, we proceed to prove the other stated estimates. They should be proved first for the approximations $\left(u^{k}, h^{k}\right)$ and then carried to $(u, h)$ in the limit. Since that is a standard procedure and the computations are exactly the same, to ease the notation we will work directly with $(u, h)$ in the rest of the paper. The technique of using exponentials as weighting functions in time was inspired by Heywood and Rannacher [6].

Multiplying the inequality (3.2) by $e^{\theta t}, \theta>0$, and integrating in time from 0 to $t$ yields

$$
\begin{gathered}
e^{\theta t}\left(\alpha\|\nabla u(t)\|^{2}+\|\nabla h(t)\|^{2}\right)+v \int_{0}^{t} e^{\theta s}\|A u(s)\|^{2} d s+\gamma \int_{0}^{t} e^{\theta s}\|A h(s)\|^{2} d s \\
\leq \alpha\left\|\nabla u_{0}\right\|^{2}+\left\|\nabla h_{0}\right\|^{2}+C_{1} \alpha^{5} \int_{0}^{t} e^{\theta s}\|\nabla u(s)\|^{10} d s+C_{1} \int_{0}^{t} e^{\theta s}\|\nabla h(s)\|^{10} d s \\
+\alpha \theta \int_{0}^{t} e^{\theta s}\|\nabla u(s)\|^{2} d s+\theta \int_{0}^{t} e^{\theta s}\|\nabla h(s)\|^{2} d s+C_{3} \int_{0}^{t} e^{\theta s} d s .
\end{gathered}
$$

On multiplying by $e^{-\theta t}$ and recalling that $\|\nabla u(t)\|$ and $\|\nabla h(t)\|$ are uniformly bounded, we get that $e^{-\theta t} \int_{0}^{t} e^{\theta s}\|A u(s)\|^{2} d s$ and $e^{-\theta t} \int_{0}^{t} e^{\theta s}\|A h(s)\|^{2} d s$ are also uniformly bounded. Setting $v=u_{t}$ in (2.1) (actually, setting $v=u_{r}^{k}$ in (2.3)), yields

$$
\alpha\left\|u_{t}\right\|^{2}=\alpha\left(f, u_{t}\right)+\left(h . \nabla h, u_{t}\right)-\alpha\left(u . \nabla u, u_{t}\right)-v\left(A \mu, u_{t}\right) .
$$


From this, we have

$$
\int_{0}^{t} e^{\theta s}\left\|u_{t}(s)\right\|^{2} d s \leq C \int_{0}^{t} e^{\theta s}\left(\|f(s)\|^{2}+\|h . \nabla h\|^{2}+\|u . \nabla u\|^{2}+\|A u\|^{2}\right) d s .
$$

Now, bearing in mind (2.4) and the Sobolev embedding $H^{2} \hookrightarrow L^{\infty}$, the following estimate is obtained.

$$
\|u . \nabla u\|^{2} \leq\|u\|_{L^{\infty}}^{2}\|\nabla u\|^{2} \leq C\|A u\|^{2}\|\nabla u\|^{2} \leq C M\|A u\|^{2} .
$$

Similarly, we have

$$
\|h . \nabla h\|^{2} \leq C M\|A h\|^{2} .
$$

Using these estimates in (3.3) produces

$$
e^{-\theta s} \int_{0}^{t} e^{\theta s}\left\|u_{t}(s)\right\|^{2} d s \leq C(M) e^{-\theta s} \int_{0}^{t} e^{\theta s}\left(\|f\|^{2}+\|A u\|^{2}+\|A h\|^{2}\right) d s .
$$

Consequently, by (2.6) together with the fact $f \in L^{\infty}\left([0, \infty), L^{2}(\Omega)\right)$, we conclude the desired estimate for $u_{t}$. The estimate for $h_{t}$ is proved in a similar way. Observe that the previous estimates hold true for $\theta \geq 0$ if we are considering finite time intervals $[0, T], 0<T<+\infty$ (with the suprema obviously depending on $T$ ). This comes from the method of proof. Thus, in a finite interval $[0, T]$, one can take the last estimates with $\theta=0$.

REMARK. As in the end of the previous proof, observe that all these estimates hold true for $\theta=0$ on the time interval $[0, \infty)$ if we also include in the hypothesis $f \in L^{2}\left([0, \infty) ; L^{2}(\Omega)\right)$.

PROOF OF THEOREM 5. To prove Theorem 5 further estimates for $u, h$ (actually $u^{k}$ and $h^{k}$ ) are needed. To this end, differentiate (2.1) (i) and (ii) (actually (2.3) (i) and (ii)) with respect to $t$ and set $\phi=u_{t}$ and $\psi=h_{t}$ (actually $\phi=u_{t}^{k}$ and $\psi=h_{t}^{k}$ ). We are left with

$$
\begin{aligned}
& \frac{\alpha}{2} \frac{d}{d t}\left\|u_{t}\right\|^{2}+v\left\|\nabla u_{t}\right\|^{2}=\alpha\left(f_{t}, u_{t}\right)-\alpha\left(u_{t} . \nabla u, u_{t}\right)+\left(h_{t} . \nabla h, u_{t}\right)+\left(h . \nabla h_{t}, u_{t}\right), \\
& \frac{1}{2} \frac{d}{d t}\left\|h_{t}\right\|^{2}+\gamma\left\|\nabla h_{t}\right\|^{2}=-\left(u_{t} . \nabla h, h_{t}\right)+\left(h_{t} . \nabla u, h_{t}\right)+\left(h . \nabla u_{t}, h_{t}\right),
\end{aligned}
$$

since $\left(u . \nabla u_{t}, u_{t}\right)=\left(u . \nabla h_{t}, h_{t}\right)=0$.

Adding the above inequalities produces

$$
\begin{aligned}
& \frac{1}{2} \frac{d}{d t}\left(\alpha\left\|u_{t}\right\|^{2}+\left\|h_{t}\right\|^{2}\right)+v\left\|\nabla u_{t}\right\|^{2}+\gamma\left\|\nabla h_{t}\right\|^{2} \\
& \quad=\alpha\left(f_{t}, u_{t}\right)-\alpha\left(u_{t} . \nabla u, u_{t}\right)+\left(h_{t} . \nabla h, u_{t}\right)-\left(u_{t} . \nabla h, h_{t}\right)+\left(h_{t} . \nabla u, h_{t}\right),
\end{aligned}
$$


since $\left(h . \nabla h_{t}, u_{t}\right)+\left(h . \nabla u_{t}, h_{t}\right)=0$. From the Sobolev-type inequality

$$
\|\varphi\|_{L^{4}} \leq\|\varphi\|^{1 / 4}\|\nabla \varphi\|^{3 / 4},
$$

we have

$$
\begin{aligned}
\left|\alpha\left(u_{t} . \nabla u, u_{t}\right)\right| & \leq \alpha\|\nabla u\|\left\|u_{t}\right\|_{L^{4}}^{2} \\
& \leq \alpha\|\nabla u\|\left\|u_{t}\right\|^{1 / 2}\left\|\nabla u_{t}\right\|^{3 / 2} \\
& \leq C\|\nabla u\|^{4}\left\|u_{t}\right\|^{2}+\frac{1}{8} v\left\|\nabla u_{t}\right\|^{2}
\end{aligned}
$$

and

$$
\begin{aligned}
\left|\left(h_{t} . \nabla h, u_{t}\right)\right| & \leq\left\|h_{t}\right\|_{L^{4}}\|\nabla h\|\left\|u_{t}\right\|_{L^{4}} \\
& \leq C\left\|h_{t}\right\|_{L^{4}}\|\nabla h\|\left\|\nabla u_{t}\right\| \\
& \leq C\left\|h_{t}\right\|_{L^{4}}^{2}\|\nabla h\|^{2}+\frac{1}{8} v\left\|\nabla u_{t}\right\|^{2} \\
& \leq C\|\nabla h\|^{2}\left\|h_{t}\right\|^{1 / 2}\left\|\nabla h_{t}\right\|^{3 / 2}+\frac{1}{8} v\left\|\nabla u_{t}\right\|^{2} \\
& \leq C\|\nabla h\|^{8}\left\|h_{t}\right\|^{2}\left\|+\frac{1}{6} \gamma\right\| \nabla h_{t}\left\|^{2}+\frac{1}{8} \nu\right\| \nabla u_{t} \|^{2} .
\end{aligned}
$$

Analogously, one can prove

$$
\begin{aligned}
& \left|\left(u_{t} \cdot \nabla h, h_{t}\right)\right| \leq C\|\nabla h\|^{8}\left\|h_{t}\right\|^{2}+\frac{1}{6} \gamma\left\|\nabla h_{t}\right\|^{2}+\frac{1}{8} \nu\left\|\nabla u_{t}\right\|^{2}, \\
& \left|\left(h_{t}, \nabla u, h_{t}\right)\right| \leq C\|\nabla u\|^{4}\left\|h_{t}\right\|^{2}+\frac{1}{6} \gamma\left\|\nabla h_{t}\right\|^{2} .
\end{aligned}
$$

Thus, by using the above inequalities in (3.4), one obtains

$$
\frac{d}{d t}\left(\alpha\left\|u_{t}\right\|^{2}+\left\|h_{t}\right\|^{2}\right)+v\left\|\nabla u_{t}\right\|^{2}+\gamma\left\|\nabla h_{t}\right\|^{2} \leq C(M)\left(\left\|u_{t}\right\|^{2}+\left\|h_{t}\right\|^{2}\right)+C\left\|f_{t}\right\|^{2} .
$$

Multiplying the above inequality by $e^{\theta t}$ and integrating the resulting inequality from 0 to $t$ produces

$$
\begin{aligned}
e^{\theta t}\left(\alpha\left\|u_{t}(t)\right\|^{2}+\left\|h_{t}(t)\right\|^{2}\right)+\int_{0}^{t} e^{\theta s}\left(\nu\left\|\nabla u_{t}(s)\right\|^{2}+\gamma\left\|\nabla h_{t}(s)\right\|^{2}\right) d s \\
\leq C(M) \int_{0}^{t} e^{\theta s}\left(\left\|u_{t}(s)\right\|^{2}+\left\|h_{t}(s)\right\|^{2}\right) d s+C \int_{0}^{t} e^{\theta s}\left\|f_{t}(s)\right\|^{2} d s \\
\quad+\alpha\left\|u_{t}(0)\right\|^{2}+\left\|h_{t}(0)\right\|^{2}+\theta \int_{0}^{t} e^{\theta s}\left(\alpha\left\|u_{t}(s)\right\|^{2}+\left\|h_{t}(s)\right\|^{2}\right) d s .
\end{aligned}
$$


Multiplying the above inequality by $e^{-\theta t}$ gives

$$
\begin{aligned}
\alpha\left\|u_{t}(t)\right\|^{2}+\left\|h_{t}(t)\right\|^{2}+e^{-\theta t} \int_{0}^{t} e^{\theta s}\left(\nu\left\|\nabla u_{t}(s)\right\|^{2}+\gamma\left\|\nabla h_{t}(s)\right\|^{2}\right) d s \\
\leq C(M) e^{-\theta t} \int_{0}^{t} e^{\theta s}\left(\left\|u_{t}(s)\right\|^{2}+\left\|h_{t}(s)\right\|^{2}\right) d s+C e^{-\theta t} \int_{0}^{t} e^{\theta s}\left\|f_{t}(s)\right\|^{2} d s \\
\quad+\alpha e^{-\theta t}\left\|u_{t}(0)\right\|^{2}+e^{-\theta t}\left\|h_{t}(0)\right\|^{2}+\theta e^{-\theta t} \int_{0}^{t} e^{\theta s}\left(\alpha\left\|u_{t}(s)\right\|^{2}+\left\|h_{t}(s)\right\|^{2}\right) d s \\
\leq C+\alpha e^{-\theta t}\left\|u_{t}(0)\right\|^{2}+e^{-\theta t}\left\|h_{t}(0)\right\|^{2},
\end{aligned}
$$

thanks to the previous estimates. So, it is enough to find estimates for $\left\|u_{t}(0)\right\|^{2}$ and $\left\|h_{t}(0)\right\|^{2}$ (actually $\left\|u_{t}^{k}(0)\right\|$ and $\left.\left\|h_{t}^{k}(0)\right\|\right)$.

For this, recall that $u_{0}, h_{0}\left(u_{0}^{k} ; h_{0}^{k}\right) \in V \cap H^{2}(\Omega)$. Consequently, setting $\phi=u_{t}$ and $\psi=h_{t}$ in (2.1) (actually $\phi=u_{t}^{k}$ and $\psi=h_{t}^{k}$ in (2.3)), yields

$$
\begin{aligned}
\alpha\left\|u_{t}\right\|^{2} & =\alpha\left(f, u_{t}\right)-v\left(A u, u_{t}\right)-\alpha\left(u . \nabla u, u_{t}\right)+\left(h . \nabla h, u_{t}\right), \\
\left\|h_{t}\right\|^{2} & =\left(h . \nabla u, h_{t}\right)-\left(u . \nabla h, h_{t}\right)-\gamma\left(A h, h_{t}\right) .
\end{aligned}
$$

The above inequalities imply

$$
\begin{aligned}
\alpha\left\|u_{t}(0)\right\| & \leq \alpha\|f(0)\|+\nu\left\|A u_{0}\right\|+\alpha\left\|A u_{0}\right\|\left\|\nabla u_{0}\right\|+\left\|A h_{0}\right\|\left\|\nabla h_{0}\right\| \leq C<+\infty, \\
\left\|h_{t}(0)\right\| & \leq\left\|A h_{0}\right\|\left\|\nabla u_{0}\right\|+\left\|A u_{0}\right\|\left\|\nabla h_{0}\right\|+\gamma\left\|A h_{0}\right\| \leq C<+\infty .
\end{aligned}
$$

Taking $\phi=A u$ and $\psi=A h$ in (2.1) (actually $\phi=A u^{k}$ and $\psi=A h^{k}$ in (2.3)) produces

$$
\begin{aligned}
v\|A u\|^{2} & \leq C\left(\|f\|^{2}+\left\|u_{t}\right\|^{2}+\|u \cdot \nabla u\|^{2}+\|h . \nabla h\|^{2}\right), \\
\gamma\|A h\|^{2} & \leq C\left(\left\|h_{t}\right\|^{2}+\|u . \nabla h\|^{2}+\|h . \nabla u\|^{2}\right) .
\end{aligned}
$$

Observe that

$$
\begin{aligned}
\|u . \nabla u\|^{2} & \leq\|u\|_{L^{4}}^{2}\|\nabla u\|_{L^{4}}^{2} \leq C\|\nabla u\|\left(\|\nabla u\|^{1 / 2}\|A u\|^{3 / 2}\right) \\
& \leq C\|\nabla u\|^{5 / 2}\|A u\|^{3 / 2} \leq C\|\nabla u\|^{10}+\frac{1}{4} v\|A u\|^{2} .
\end{aligned}
$$

Analogously, we obtain

$$
\begin{aligned}
\|h . \nabla h\| & \leq C\|\nabla h\|^{10}+\frac{1}{4} \gamma\|A h\|^{2}, \\
\|u . \nabla h\|^{2} & \leq C\|\nabla u\|^{8}\|\nabla h\|^{2}+\frac{1}{4} \gamma\|A h\|^{2}, \\
\|h . \nabla u\|^{2} & \leq C\|\nabla h\|^{8}\|\nabla u\|^{2}+\frac{1}{4} v\|A u\|^{2} .
\end{aligned}
$$


Now, adding inequalities (3.5) and (3.6) and using the above estimates, produces

$$
\begin{aligned}
v\|A u\|^{2}+\gamma\|A h\|^{2} \leq & C\left(\|f\|^{2}+\left\|u_{t}\right\|^{2}+\left\|h_{t}\right\|^{2}+\|\nabla u\|^{10}+\|\nabla h\|^{10}\right. \\
& \left.+\|\nabla u\|^{8}\|\nabla h\|^{2}+\|\nabla h\|^{8}\|\nabla u\|^{2}\right) \\
\leq C & <+\infty .
\end{aligned}
$$

Thanks to the previous estimates, this implies sup $\|A u(t)\|^{2} \leq C$, sup $\|A h(t)\|^{2} \leq C$.

Differentiating (2.1) (i) (actually (2.5) (i) with respect to $t$ ) yields

$$
\alpha u_{t t}=P\left(\alpha f_{t}+h_{t} . \nabla h+h \cdot \nabla h_{t}-\alpha u_{t} \cdot \nabla u-\alpha u . \nabla u_{t}\right)-v A u_{t} \equiv g .
$$

Consequently,

$$
e^{-\theta t} \int_{0}^{t} e^{\theta t}\left\|u_{t t}\right\|_{V^{*}}^{2} d s \leq e^{-\theta t} \int_{0}^{t} e^{\theta s}\|g\|_{V^{*}}^{2} d s .
$$

This is sufficient to estimate the right-hand side. To do so, observe that

$$
\begin{aligned}
\left\|P\left(u_{t}, \nabla h\right)\right\|_{v^{*}} & =\sup _{\|v\|_{v} \leq 1}\left|\left(u_{t}, \nabla h, v\right)\right| \\
& \leq C \sup _{\|v\| v \leq 1}\left\|\nabla u_{t}\right\|\|\nabla h\|\|\nabla v\| \\
& \leq C\|\nabla h\|\left\|\nabla u_{t}\right\| \leq C M\left\|\nabla u_{t}\right\| .
\end{aligned}
$$

Consequently, for all $t \geq 0$,

$$
e^{-\theta t} \int_{0}^{t} e^{\theta s}\left\|P\left(u_{t} . \nabla h\right)\right\|_{V}^{2} \cdot d s \leq C M^{2} e^{-\theta t} \int_{0}^{t} e^{\theta s}\left\|\nabla u_{t}\right\|^{2} d s \leq C,
$$

thanks to estimate (2.9). Also,

$$
\left\|A u_{t}\right\|_{v \cdot}=\sup _{\|v\|_{v} \leq 1}\left|\left(A u_{t}, v\right)\right|=\sup _{\|v\|_{v} \leq 1}\left|\left(\nabla u_{t}, \nabla v\right)\right| \leq\left\|\nabla u_{t}\right\|
$$

Thus

$$
e^{-\theta t} \int_{0}^{t} e^{\theta s}\left\|A u_{t}\right\|_{v \cdot}^{2} d s \leq e^{-\theta t} \int_{0}^{t} e^{\theta s}\left\|\nabla u_{t}\right\|^{2} d s \leq C .
$$

The other terms in (3.7) are estimated analogously. We obtain

$$
\sup _{t \geq 0} e^{-\theta t} \int_{0}^{t} e^{\theta s}\left\|u_{t t}\right\|_{V}^{2} \cdot d s \leq \sup _{t \geq 0} e^{-\theta t} \int_{0}^{t} e^{\theta s}\|g(s)\|_{V}^{2} \cdot d s \leq C
$$

and this completes the proof of the theorem. 


\section{The proofs of Theorems 6 and 7}

The remarks made at the beginning of the proof of Theorem 4 also hold here. Working as in Boldrini and Rojas-Medar [2, p. 6], we have

$$
\begin{aligned}
& \frac{\alpha}{2} \frac{d}{d t}\|u\|^{2}+\nu\|\nabla u\|^{2}=\alpha(f, u)+(h . \nabla h, u), \\
& \frac{1}{2} \frac{d}{d t}\|h\|^{2}+\gamma\|\nabla h\|^{2}=(h . \nabla u, h) .
\end{aligned}
$$

Adding the above identities gives

$$
\frac{d}{d t}\left(\alpha\|u\|^{2}+\|h\|^{2}\right)+2 v\|\nabla u\|^{2}+2 \gamma\|\nabla h\|^{2}=2 \alpha(f, u) \leq C\|f\|^{2}+\nu\|\nabla u\|^{2} .
$$

By multiplying the above equation by $e^{\bar{\theta} t}$ with $\bar{\theta}>0$ to be chosen later on, we conclude that

$$
\frac{d}{d t} e^{\bar{\theta}_{t}}\left(\alpha\|u\|^{2}+\|h\|^{2}\right)+v e^{\bar{\theta} t}\|\nabla u\|^{2}+2 \gamma e^{\overline{\theta_{t}}}\|\nabla h\|^{2} \leq C e^{\bar{\theta} t}\|f\|^{2}+\bar{\theta} e^{\bar{\theta} t}\left(\alpha\|u\|^{2}+\|h\|^{2}\right) .
$$

Since $\|\phi\|^{2} \leq C_{\Omega}\|\nabla \phi\|^{2}$ for $\phi \in H_{0}^{1}(\Omega)$, we have

$$
\frac{d}{d t} e^{\bar{\theta} t}\left(\alpha\|u\|^{2}+\|h\|^{2}\right)+C e^{\bar{\theta} t}\left(\|\nabla u\|^{2}+\|\nabla h\|^{2}\right) \leq C e^{\bar{\theta} t}\|f\|^{2}
$$

for $0<\bar{\theta}<\min \left\{2 \gamma / C_{\Omega}, v / \alpha C_{\Omega}\right\}$.

The above inequality implies

$$
\begin{gathered}
\alpha\|u(t)\|^{2}+\|h(t)\|^{2}+C e^{-\bar{\theta} t} \int_{0}^{t} e^{\bar{\theta} s}\left(\|\nabla u(s)\|^{2}+\|\nabla h(s)\|^{2}\right) d s \\
\leq e^{-\bar{\theta} t}\left(\alpha\left\|u_{0}\right\|^{2}+\left\|h_{0}\right\|^{2}\right)+C e^{-\bar{\theta}_{t}} \int_{0}^{t} e^{\bar{\theta} s}\|f(s)\|^{2} d s \\
\leq \alpha\left\|u_{0}\right\|^{2}+\left\|h_{0}\right\|^{2}+C\|f\|_{L^{\infty}}^{2} \leq C<+\infty .
\end{gathered}
$$

Taking $\phi=A u$ and $\psi=A h$ in (2.1), gives

$$
\begin{aligned}
\frac{\alpha}{2} \frac{d}{d t}\|\nabla u\|^{2}+v\|A u\|^{2} & =\alpha(f, A u)+(h . \nabla h, A u)-\alpha(u . \nabla u, A u), \\
\frac{1}{2} \frac{d}{d t}\|\nabla h\|^{2}+\gamma\|A h\|^{2} & =(h . \nabla u, A h)-(u . \nabla h, A h) .
\end{aligned}
$$

Recall the following inequality that is only true in the two-dimensional case (see $[8$, p. 70])

$$
\|\phi\|_{L^{4}} \leq C\|\nabla \phi\|^{1 / 2}\|\phi\|^{1 / 2} .
$$


Using this inequality produces

$$
\begin{aligned}
|(h . \nabla u, A h)| & \leq\|h\|_{L^{4}}\|\nabla u\|_{L^{4}}\|A h\| \\
& \leq C_{\varepsilon}\|h\|_{L^{4}}^{2}\|\nabla u\|_{L^{4}}^{2}+\varepsilon\|A h\|^{2} \\
& \leq C_{\varepsilon}\|\nabla h\|\|h\|\|A u\|\|\nabla u\|+\varepsilon\|A h\|^{2} \\
& \leq C_{\varepsilon, \delta}\|h\|^{2}\|\nabla h\|^{2}\|\nabla u\|^{2}+\varepsilon\|A h\|^{2}+\delta\|A u\|^{2} .
\end{aligned}
$$

Analogously,

$$
\begin{aligned}
& |(u . \nabla u, A u)| \leq C_{\delta}\|\nabla u\|^{4}+\delta\|A u\|^{2}, \\
& |(u . \nabla h, A h)| \leq C_{\varepsilon}\|u\|^{2}\|\nabla u\|^{2}\|\nabla h\|^{2}+\varepsilon\|A h\|^{2}, \\
& |(h . \nabla h, A u)| \leq C_{\varepsilon, \delta}\|h\|^{2}\|\nabla h\|^{4}+\varepsilon\|A h\|^{2}+\delta\|A u\|^{2} .
\end{aligned}
$$

Adding the identities (4.1) and (4.2) and using the above estimates, with suitably chosen $\varepsilon$ and $\delta$, yields

$$
\begin{aligned}
& \frac{d}{d t}\left(\alpha\|\nabla u\|^{2}+\|\nabla h\|^{2}\right)+v\|A u\|^{2}+\gamma\|A h\|^{2} \\
& \quad \leq C\left(\|\nabla h\|^{2}\|\nabla u\|^{2}+\|\nabla u\|^{4}+\|\nabla h\|^{4}\right)+C\|f\|^{2} .
\end{aligned}
$$

In particular,

$$
\frac{d}{d t}\left(\alpha\|\nabla u\|^{2}+\|\nabla h\|^{2}\right) \leq C\left(\alpha\|\nabla u\|^{2}+\|\nabla h\|^{2}\right)^{2}+C\|f\|^{2} .
$$

Setting $\psi(t)=\alpha\|\nabla u(t)\|^{2}+\|\nabla h(t)\|^{2}$ in the last inequality produces

$$
\frac{d}{d t} \psi \leq C \psi^{2}+C\|f\|^{2}
$$

Observe that $C \psi^{2}+C_{1} \leq 2 C \psi^{2}$ for $\psi \geq\left(C_{1} / C\right)^{1 / 2}$, where $C_{1}=C \sup _{t \in[0, T]}\|f(t)\|$. If $\ell^{*}=\max \left\{\left(C_{1} / C\right)^{1 / 2}, 1, \alpha\left\|\nabla u_{0}\right\|^{2}+\left\|\nabla h_{0}\right\|^{2}\right\}$, then either $0 \leq \psi(t) \leq \ell^{*}$ for all $t \geq 0$ or there exists some interval $\left[t_{1}, t_{2}\right], t_{2}>t_{1}$ for which $\alpha\left\|\nabla u\left(t_{1}\right)\right\|^{2}+\left\|\nabla h\left(t_{1}\right)\right\|^{2}=$ $\ell^{*}$ and for $t \in\left[t_{1}, t_{2}\right]$ it is true that $\alpha\|\nabla u(t)\|^{2}+\|\nabla h(t)\|^{2} \geq \ell^{*}$. Then, due to the choice of $\ell^{*}$, in this interval $\left[t_{1}, t_{2}\right], \frac{d}{d t} \psi \leq C \psi^{2}$ holds or, equivalently,

$$
\frac{d}{d t} \ln \psi \leq C \psi
$$

Multiplying the above inequality by $e^{\bar{\theta} t}$ gives

$$
\frac{d}{d t} e^{\bar{\theta} t} \ln \psi \leq C e^{\bar{\theta} t} \psi+\bar{\theta} e^{\bar{\theta} t} \ln \psi
$$


Observe that there exists a positive constant $\rho$ such that $\ln \psi \leq \rho+\rho \psi$. Using this and integrating the last inequality from $t_{1}$ to $t \in\left[t_{1}, t_{2}\right]$ gives

$$
e^{\bar{\theta} t} \ln \psi(t)-e^{\bar{\theta} t_{1}} \ln \psi\left(t_{1}\right) \leq(C+\bar{\theta} \rho) \int_{0}^{t} e^{\bar{\theta} s} \psi(s) d s+\bar{\theta} \rho \int_{t_{1}}^{t} e^{\bar{\theta} s} d s .
$$

Hence,

$$
\begin{aligned}
\ln \psi(t)-e^{\bar{\theta}\left(t_{1}-t\right)} \ln \psi\left(t_{1}\right) & \leq(C+\bar{\theta} \rho) e^{-\bar{\theta} t} \int_{t_{1}}^{t} e^{\bar{\theta} s} \psi(s) d s+\bar{\theta} \rho e^{-\bar{\theta} t} \int_{t_{1}}^{t} e^{\bar{\theta} s} d s \\
& \leq(C+\bar{\theta} \rho)\left[\alpha\left\|u_{0}\right\|^{2}+\left\|h_{0}\right\|^{2}+\|f\|_{L^{\infty}}^{2}\right]+\rho\left[1-e^{\bar{\theta} t_{2}}\right] \\
& \leq(C+\bar{\theta} \rho)\left[\alpha\left\|u_{0}\right\|^{2}+\left\|h_{0}\right\|^{2}+\|f\|_{L^{\infty}}^{2}\right]+\rho \equiv \bar{M} .
\end{aligned}
$$

Consequently, since $-e^{\bar{\theta}\left(t_{1}-t\right)} \ln \psi\left(t_{1}\right) \geq-\ln \psi\left(t_{1}\right)$, we have $\ln \left(\psi(t) / \psi\left(t_{1}\right)\right) \leq \bar{M}$, which implies that for all $t \in\left[t_{1}, t_{2}\right]$

$$
\alpha\|\nabla u(t)\|^{2}+\|\nabla h(t)\|^{2} \leq\left(\alpha\left\|\nabla u\left(t_{1}\right)\right\|^{2}+\left\|\nabla h\left(t_{1}\right)\right\|^{2}\right) e^{\bar{M}}=\ell^{*} e^{\bar{M}} .
$$

Since this is independent of $t_{1}$ and $t_{2}$, we conclude that for all $t \geq 0$

$$
\alpha\|\nabla u(t)\|^{2}+\|\nabla h(t)\|^{2} \leq \max \left\{\ell^{*}, \ell^{*} e^{\bar{M}}\right\} \equiv \ell^{*} e^{\bar{M}} .
$$

The rest of analysis is now done exactly as in the three-dimensional case.

\section{Proof of Theorem 8}

Observe that (2.1) (i) and (2.1) (ii) are equivalent to $A u=P(F)$ and $A h=P(G)$, respectively, where $F=\alpha f-\alpha u_{t}-\alpha u . \nabla u+h . \nabla h$ and $G=h . \nabla u-u . \nabla h-h_{t}$.

Under the hypothesis of Theorem 4 or 6 (respectively of Theorem 5 or 7), we have $F, G \in L_{l o c}^{2}\left(0, \infty ; L^{2}(\Omega)\right)$ (respectively, $F, G \in L^{\infty}\left(0, \infty ; L^{2}(\Omega)\right)$ ).

Therefore, Amrouche and Girault's results [1] imply that there are unique $p, w \in$ $L_{l o c}^{2}\left(0, \infty ; H^{1}(\Omega) / \mathbb{R}\right)$ (respectively, $p, w \in L^{\infty}\left(0, \infty ; H^{1}(\Omega) / \mathbb{R}\right) \cap C([0, \infty) ;$ $\left.L^{2}(\Omega) / \mathbb{R}\right)$ such that

$$
-v \Delta u+\nabla p=F, \quad \operatorname{div} u=0,\left.\quad u\right|_{\Gamma}=0
$$

and

$$
-\gamma \Delta h+\nabla w=G, \quad \operatorname{div} h=0,\left.\quad h\right|_{\Gamma}=0 .
$$

It is enough to take $p^{*}=p-\frac{\mu}{2} h^{2}$ and Theorem 8 is proved. Estimates (2.11) (respectively (2.12)) follow easily from the previous estimate and the estimates given in the above section. This completes the proof of the theorem. 


\section{References}

[1] G. Amrouche and V. Girault, "On the existence and regularity of the solutions of Stokes problem in arbitrary dimension", Proc. Japan Acad., Ser. A 67 (1991) 171-175.

[2] J. L. Boldrini and M. Rojas-Medar, "Global strong solutions of the equations for the motion of nonhomogeneous incompressible fluids", in Research Report 1993/23, IMECC-UNICAMP, (Universidade Estadual de Campinas, Brazil, 1993).

[3] E. V. Chizhonkov, "On a system of equations of magnetohydrodynamic type", Soviet Math. Dokl. 30 (1984) 542-545.

[4] P. Constantin and C. Foias, Navier-Stokes equations (The University of Chicago Press, Chicago, 1988).

[5] H. Fujita and T. Kato, "On the Navier-Stokes initial value problem, I", Arch. Rational Mech. Anal. 16 (1964) 269-315.

[6] J. G. Heywood and R. Rannacher, "Finite element approximation of the nonstationary NavierStokes problem I: regularity of solutions and second order error estimates for spatial discretization", SIAM J. Num. Anal. 19 (1982) 275-311.

[7] G. Lassner, "Über ein rand-anfangswert-problem der magnetohydrodinamik", Arch. Rational Mech. Anal. 25 (1967) 388-405.

[8] J. L. Lions, Quelques méthodes de résolution des problémes aux limites non-linéares (Dunod Gauthier-Villars, Paris, 1969).

[9] S. B. Pikelner, Grundlagen der Kosmischen Elektrodynamik (Moskau, 1966).

[10] A. Schlüter, "Dynamik des Plasmas I", Z. Naturforsch. 5a. (1950) 72-78.

[11] A. Schlüter, "Dynamik des Plasmas II", Z. Naturforsch. 6a. (1951) 73-79.

[12] R. Temam, Navier-Stokes equations (North-Holland, Amsterdam, 1979). 